\title{
Impact of mushy frozen water particles onto a cold surface
}

\author{
Mark Gloerfeld*, Markus Schremb, llia V. Roisman, Cameron Tropea , Jeanette Hussong \\ Institute of Fluid Mechanics and Aerodynamics, Technical University Darmstadt, Darmstadt, \\ Germany \\ *Corresponding author email: gloerfeld@sla.tu-darmstadt.de
}

\begin{abstract}
Icing, as a result from the impact of supercooled water drops, is a hazard for structures exposed to low temperatures, for instance aircraft and wind turbines. Despite very intensive study of the involved phenomena, the underlying physical processes are not yet entirely understood; hence, modelling of the conditions for ice accretion and prediction of the icing rate are presently not reliable. A major difference between solidification of a liquid drop below the melting temperature and a supercooled drop is a very fast dendritic solidification phase associated with the latter. In the present experimental study, the impact behaviour of such mushy, dendritic drops is investigated using a high-speed video system. The impact parameters and the initial liquid drop temperature are varied in the experiments in order to characterize particle deformation and its residual shape. The dynamics of impact of such particles have never been studied before.

A theoretical impact model for the particle deformation is applied to this problem. The model is based on the assumption that the material properties of mushy particles can be approximated by rigid-perfectly plastic behaviour. Specifically the yield strength of such particles is estimated as a function of solid ice content, which is estimated for different initial temperatures of particles prior to freezing.
\end{abstract}

\section{Keywords}

Particle impact, Icing, Supercooled drops, Dendritic freezing

\section{Introduction}

Icing of structures through the impact of liquid drops is a serious safety and operational hazard for many exposed structures, including aircraft [1], wind turbines [2], high-voltage transmission lines [3], ships [4] etc. Therefore, there is a strong demand for reliable prediction of impact probability (capture efficiency), impact dynamics, including deposition ratio, secondary drop splashing behaviour and ice accretion, as well as shedding scenarios. The current computational codes for ice accretion based on existing models are plagued by a very high degree of empiricism which leads to little universality of correlations and unsatisfactory reliability of predictions. Therefore, there exists considerable demand to introduce a stronger degree of physics into existing models and subsequently into predictive codes.

The impact of pure liquid drops onto surfaces has enjoyed tremendous attention in the scientific community over the past few decades, in part due to the availability of affordable high-speed cameras. A comprehensive review of these studies can be found in [5-7] and elswhere. However, in icing scenarios the possibility of nucleation and solidification must be accounted for. These processes take on a very special character when dealing with supercooled liquid drops, which are very common in most of the icing events referred to above.

The solidification of a supercooled drop includes two main phases [8-10]. In the first phase, an initial nucleus promotes the growth of dendrites, which propagate throughout the liquid bulk extremely fast, releasing heat into the surrounding liquid in the form of latent heat of solidification. After this phase, the drop can be considered as a mushy composition of solid dendrites surrounded by liquid water at the melting temperature. The second phase of solidification follows on the dendritic freezing if the mixture is further cooled, e.g. through a wall temperature 
initially being significantly lower than the melting temperature. This phase represents a stable, planar, relatively slow freezing of the liquid phase of the mushy region, which is described by the Stefan law.

The dendritic frozen state can occur after drop impact due to heterogeneous nucleation arising from disturbances related to the liquid-substrate contact and the unique hydrodynamic features of drop impact [11]. However, the possibility also exists that nucleation takes place prior to impact; a very real possibility in the atmosphere, where contaminants in the form of particles are present. It is therefore not unlikely that drops impacting onto surfaces are not pure liquid, but drops in the dendritic frozen state. Clearly, these will exhibit a different impact behaviour than pure liquid drops, which is the subject of the present study.

To the authors' knowledge there has been no work devoted to investigating the impact of mushy particles originated from supercooled drops. Nevertheless, the interest in the impact of twophase drops in general has grown in the recent past. A review of compound drop impact is found in [12], although this work mainly deals with two liquid phases. Studies involving a solid component in the drop were, for instance, conducted by [13] who investigated the impact of a sand/water mixture drop. A more recent study from [14] examines the impact of a partially frozen binary drop consisting of hexadecane and diethyl ether. However, these drops solidify evenly from the outside to the centre during their descent.

In the present study interest is directed towards the degree of deposition after mushy particle impact, as this is considered to be instrumental in determining ice accretion rates, a particular important quantity in predicting icing related safety factors in the aircraft industry. For this reason, an experiment has been designed in which the degree of supercooling and the velocity of impacting drops onto cold surfaces can be extremely well controlled and systematically varied. The goal is first to make observations about the general outcome of impact, using high-speed video cameras. These results then lead to model formulations to describe the observations, incorporating as much physics as possible, in order to make the models more universal. In this case, an existing model formulated for ice particle impact onto surfaces has been used and adapted for the case of a mushy particle [15]. The result is a first correlation of yield strength with degree of supercooling and impact velocity. Physically, the height of the deposited ice on the surface has been measured and can be explained in terms of differing yield strength.

\section{Experimental setup}

Experiments involving impact of mushy frozen particles originated from supercooled drops have been carried out in a vertical open return wind tunnel, in which the drop trajectory is aligned with the vertical airflow. A schematic of the tunnel is depicted in Figure 1. The test section has a square cross-section with a side length of $140 \mathrm{~mm}$. The air flow in it reaches uniform air velocities across the test section from 0 to $15 \mathrm{~m} / \mathrm{s}$ with a turbulence intensity of $0.5 \%$. At the lower end of the test section a target is placed on which the mushy particle impact occurs. The entire wind tunnel is placed inside a cooling chamber, which maintains ambient temperatures of down to $-15^{\circ} \mathrm{C}$. The airflow temperature, impact target temperature as well as the drop temperature are therefore controlled entirely by the ambient conditions inside the chamber.

Water drops are generated from purified de-ionized water (Millipore, Milli- $Q^{\circledR}$ ) using a syringe needle placed inside of the wind tunnel and protected from the airflow by a shroud pipe. The temperature of the suspended drop on the syringe is continuously measured until drop detachment using a thermocouple immersed into the drop [11]. After detachment, the drop is accelerated by gravity inside of the shroud pipe until entering the airflow, where aerodynamic forces further increase drop acceleration until the drop finally impacts onto the target.

The water drops do not necessarily freeze during their detachment from the needle or their impact onto the target, as already shown in [11]; hence, nucleation is forced in the present study. For this purpose, several small ice crystals are pinned to the end of the shroud pipe. In this manner, the drop makes contact with some of the ice crystals when leaving the pipe, 


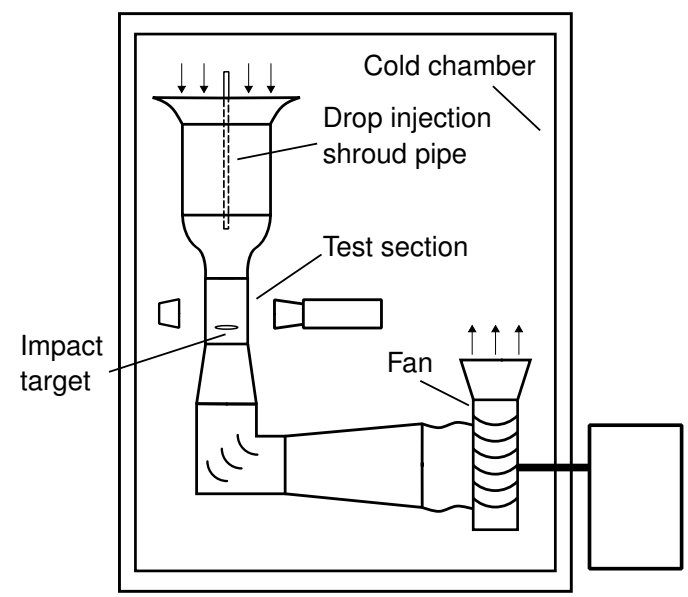

Figure 1. Icing wind tunnel setup for investigating the impact of dendritic frozen water drops.

which immediately results in dendritic freezing of the supercooled drop. Since the typical time for dendritic freezing of the drop, $\mathcal{O}(10 \mathrm{~ms})$, is much shorter than the time it takes the drop to reach the impact target, $\mathcal{O}(100 \mathrm{~ms})$ after nucleation, all drops in the present study have finished the first phase of supercooled solidification upon impact.

The drops generated in the present study initially vary in temperature (controlling its final frozen fraction) as well as in impact speed. While the temperature is indirectly controlled through the cold chamber conditions, the impact speed $U_{0}$ is varied through a varying air flow velocity. At the latest upon impact, the mushy particle has always acquired a temperature of $0^{\circ} \mathrm{C}$, since the first phase of solidification of a supercooled drop results in a mixture of ice and remaining liquid being in thermal equilibrium at the melting temperature. The initial temperature of the drop deviates at maximum $1.5^{\circ} \mathrm{C}$ from the temperature of the air flow and the impact target. $\mathrm{A}$ test matrix summarizing the experimental conditions in the present study is given in Table 1.

Table 1. Test matrix of the conducted experiments for mushy particle impact.

\begin{tabular}{c|c|c}
\hline$\Delta T_{0}(\mathrm{~K})$ & $U_{0}(\mathrm{~m} / \mathrm{s})$ & $D_{0}(\mu \mathrm{m})$ \\
\hline-11.5 to -7 & 3.8 to 5.6 & 3000
\end{tabular}

The impact of the mushy particles is captured using a high-speed video camera (Photron Fastcam $S A-X 2$ ) and backlight illumination. From the recordings, the impact dynamics as well as the residual height of the deposited ice/water mixture is obtained.

\section{Ice content of the mushy particles}

The dependence of the fraction of ice on the initial temperature of the liquid drop can be determined from the mass and energy balance during freezing $[16,17]$. Dendrite formation leads to the nearly uniform temperature of the liquid part and of the dendrites, very close to the melting point. The fraction of frozen liquid $\xi_{\text {ice }}$ after dendritic solidification is obtained from the ratio of sensible heat warming up the remaining liquid to the latent heat of fusion [18]:

$$
\xi_{\text {ice }}=\frac{\rho_{w} c_{p, w} \Delta T_{0}}{\rho_{\text {ice }} L+\Delta T\left(\rho_{w} c_{p, w}-\rho_{\text {ice }} c_{p, \text { ice }}\right)} .
$$

Here, $\Delta T_{0}$ represents the initial supercooling of the liquid and $L$ the latent heat of fusion. Furthermore, $\rho_{w}$ and $c_{p, w}$ are the density and heat capacity of the supercooled liquid respectively, and correspondingly, $\rho_{\text {ice }}$ and $c_{p}$,ice are the density and heat capacity of ice at $0^{\circ} \mathrm{C}$. The ice fraction of the mushy particles used in the experiments ranges between $\xi_{\text {ice }}\left(\Delta T_{0}=-11.5 \mathrm{~K}\right)=$ $15.0 \%$ and $\xi_{\text {ice }}\left(\Delta T_{0}=-7 \mathrm{~K}\right)=9.4 \%$. 


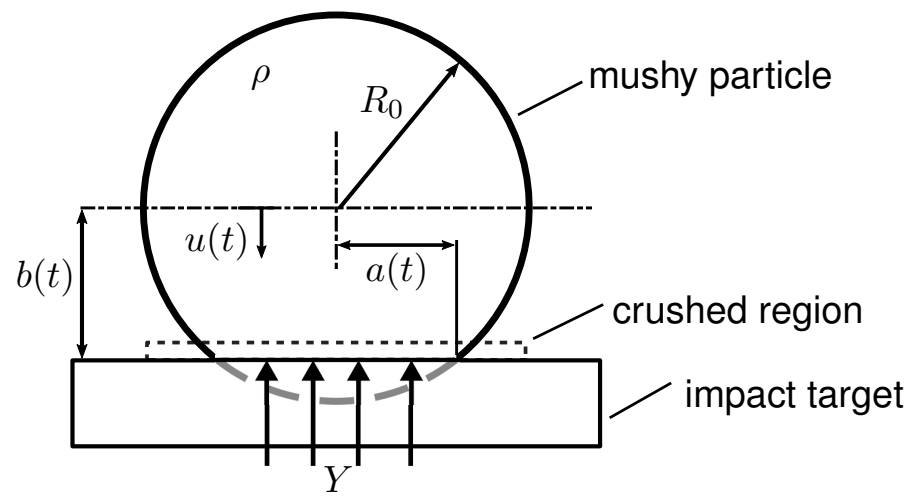

Figure 2. Kinematic and geometrical quantities of the deforming plastic mushy particle upon impact. Sketch based on the model of [15].

\section{Impact of a rigid-perfectly plastic sphere onto a rigid wall}

In order to model the impact and deformation of a mushy particle, its material behaviour is assumed to be rigid-perfectly plastic. It is characterized by a constant yield strength $Y$. A simplified quasi one-dimensional model describing the dynamics of such a sphere during the impact has been developed in [15] in the application to a problem of ice crystal impact. The model allows to predict the residual height of the particle after impact for a given yield strength. The fundamental physics and assumptions of the model are outlined in the following.

The force acting on a crushed particle with initial radius $R_{0}$ is linked to the area in contact with the surface. During impact, the particle is considered as a truncated sphere, as shown in Figure 2. The contact area of the particle is $\pi a^{2}$, where $a(t)$ is the impression radius. The impression radius changes in time due to the particle deformation. Its relation to the axial coordinate of the particle centre point $b(t)$, defined in Figure 2, can be determined from geometrical considerations. Introducing a dimensionless displacement of the drop tip $\delta(t)$ with $b(t)=R_{0}(1-\delta(t))$, we can express the impression radius $a$ in the form

$$
a(\delta)=\sqrt{R_{0}^{2}-b^{2}}=R_{0} \sqrt{(2-\delta) \delta} .
$$

Assuming that the thickness of the crushed region is much smaller than $R_{0}$, the volume of the truncated particle can be obtained from

$$
V(\delta)=\int_{-b}^{R_{0}} \pi\left(R_{0}^{2}-z^{2}\right) \mathrm{d} z=\frac{\pi R_{0}^{3}}{3}(2-\delta)^{2}(1+\delta) .
$$

The force causing shattering of the particle at the bottom is assumed to be directly linked to its yield strength $Y$. Hence, the equation of motion yields

$$
\frac{\mathrm{d}^{2} \delta}{\mathrm{d} t^{2}}=-\frac{Y \pi a(\delta)^{2}}{R_{0} \rho V(\delta)}
$$

with $\rho$ being the particle density. Considering $u \equiv \mathrm{d} b(t) / \mathrm{d} t$ and $u(t=0)=U_{0}$, along with the initial conditions

$$
\delta=0, \quad \frac{\mathrm{d} \delta}{\mathrm{d} t}=\frac{U_{0}}{R_{0}} \quad \text { at } \quad t=0,
$$

an analytical solution exists for the ordinary differential equation Eq. (4) for a constant yield strength $Y$. The expression for the velocity of the rear part of the deforming particle $u(\delta)$ is obtained in the form

$$
u(\delta)^{2}=U_{0}^{2}+\frac{2 Y}{\rho} \ln \left[\frac{(2-\delta)^{2}(1+\delta)}{4}\right] .
$$



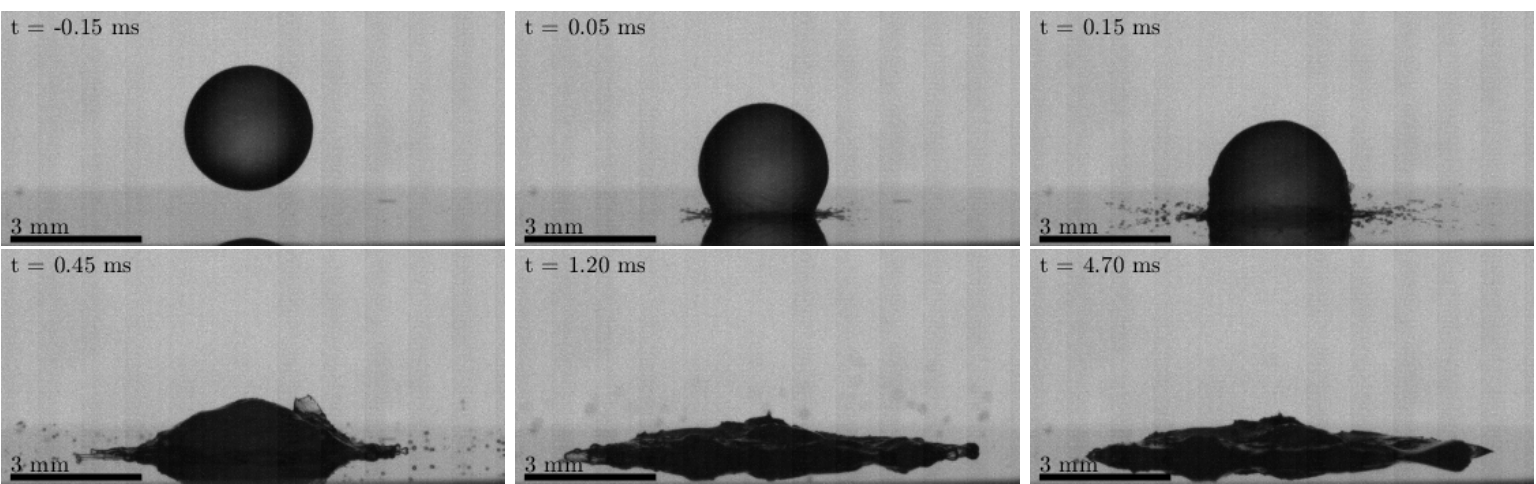

Figure 3. Image sequence of a mushy particle impacting with $U_{0}=4.2 \mathrm{~m} / \mathrm{s}$ originating from a drop with an initial supercooling of $\Delta T_{0}=-9.2 \mathrm{~K}$.

Correspondingly, the maximum displacement $\delta=\delta_{\max }$ is achieved at the instant when the velocity $u$ vanishes, finally yielding

$$
\frac{1}{Y}=-\frac{2}{\rho U_{0}^{2}} \ln \left[\frac{\left(2-\delta_{\max }\right)^{2}\left(1+\delta_{\max }\right)}{4}\right] .
$$

\section{Results and discussion}

The formation of solid ice dendrites inside the supercooled drop causes a significant change of the particle material properties and its impact behaviour. A purely liquid drop develops a thin lamella spreading over the surface as a consequence of the viscous flow of the impacting fluid [7]. In contrast, the mushy particle exhibits more of a plastic flow behaviour, resulting in a nearly cone-shaped ice agglomeration at the impact position. This behaviour is visible in the image sequence shown in Figure 3. The dendritic frozen state of the drop is already evident from the drop appearance before the impact in the first image. A purely liquid drop would exhibit a bright spot in the middle of the drop, resulting from first-order refracted light passing through the drop. However, the dendrites in the mushy particle cause a diffuse light scattering within the drop, inhibiting the undisturbed light passage through the drop.

Shortly after impact, small fragments leave the spreading mushy particle similar to a prompt splash of a liquid drop. However, the absence of glare points on them, and the size of the emitted particles, suggest a composition of water and ice, i.e. fragmented dendrites splashing away from the surface. Subsequently, the particle spreads over the surface with a noticeable deceleration of the ensuing ice/water mixture. Eventually, the mushy phase comes to rest and a cone shape agglomeration remains, as shown in the last two images of Figure 3. This agglomeration does not significantly change shape and solidification continues until all liquid is frozen.

Note, that the mushy particle locally exhibits features of both liquid and ice upon impact. At $t=0.45 \mathrm{~ms}$ (Figure 3) a small ice platelet is visible on the right side of the drop. During the impact, this ice platelet remains intact, retaining its elevated position while the remaining mixture flows away below. Finally, it remains on the surface of the resulting cone. The occurrence of this platelet suggests that nucleation had originally occurred in this region, since at this point the water remaining after dendritic freezing apparently has had enough time to further freeze to a continuous ice phase. Yet, the size of the platelet and the absence of further platelets indicate no significant influence on the behavior of other regions of the mixture.

The separation of liquid from the mixture during impact is also visible. In the last image of the impact $(t=4.7 \mathrm{~ms})$, a small amount of liquid is visible on the right side of the drop. It leaves the cone shape distinctly after the residual mixture has come to rest. This liquid movement is attributed to residual inertial forces. Notable is also that, despite the escape of fluid, the general cone shape and its residual height remain unaffected by the separation of ice or water from the 


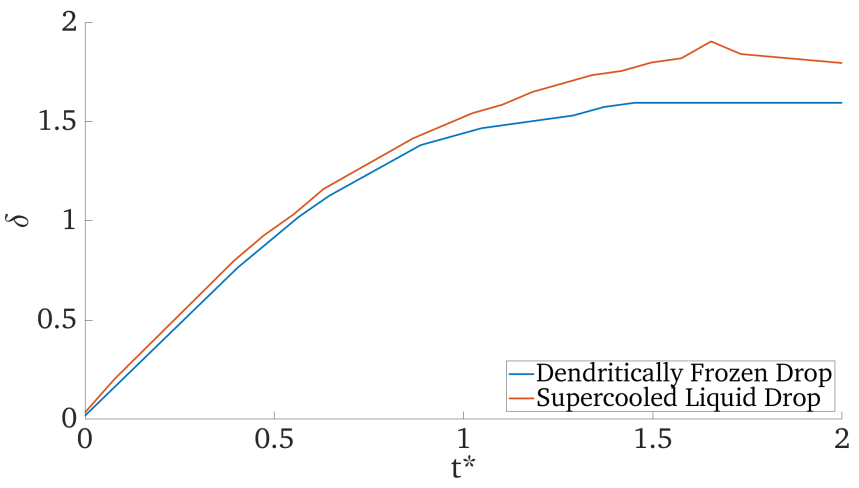

Figure 4. Comparison of the dimensionless displacement of a dendritically frozen drop (a mushy particle) and a liquid drop $\left(D=3000 \mu \mathrm{m}\right.$ and $\left.U_{0}=4.6 \mathrm{~m} / \mathrm{s}\right) . \delta$ corresponds to the dimensionless displacement of the apex of the drop and time is rendered dimensionless as $t^{*}=t U_{0} / D_{0}$. The initial supercooling of the liquid drop is

$$
\Delta T_{0}=-9.6 \mathrm{~K} \text {. }
$$

mixture.

With regard to the plastic flow behaviour and the reduction of velocity normal to the surface, the residual height is characteristic for the composition of the particles mushy phase. The velocity of the ensuing mixture is decreased noticeably, leading to a change in height progression during and after the impact. In order to outline this difference with regard to a liquid drop impact, a comparison of the temporal evolution of the apex height during impact for a mushy particle and a liquid drop is considered.

The dimensionless displacement $\delta$ depending on the dimensionless time $t^{*}$ is shown in Figure 4. The definition of $\delta$ corresponds to the model introduced above and it is related to the instantaneous height $h(t)$ by

$$
\delta(t)=2-\frac{h(t)}{R_{0}}
$$

implying $\delta\left(h=2 R_{0}\right)=0$ and $\delta(h=0)=2$.

As shown in Figure 4, during the beginning of the kinematic phase of drop impact, the height of the pure liquid drop as well as that of the ice/water mixture evolve similarly with dimensionless time. Initially the fluid at the apex of the drop remains unaffected by the fluid impact on the surface. A liquid drop continues in this manner with only minor deceleration. Its final height corresponds to the height of the rim on the periphery of the drop splat. Presumably inside the rim, the remaining liquid layer is thinner, but is obscured from view by the rim. The apex of the mushy particle exhibits a significant change in velocity shortly after $\delta \approx 1$. At this point, the ice/water mixture causes a deceleration of the drop velocity normal to the substrate. After further deceleration, the mushy particle reaches its residual height which corresponds to the height of the cone tip.

The residual height of the cone is controlled by the properties of the mushy particle investigated in this study. The yield strength is expected to depend on the fraction of solid ice in the mushy particle. The value of the yield strength can be evaluated using Eq. (7), the experimental data of the impact velocity $U_{0}$ and the maximum dimensionless displacement $\delta_{\max }$. The associated impact velocity $U_{0}$ is obtained from the high-speed recordings. The maximum displacement of the mushy particle after its impact is obtained with the help of Eq. (8) and the experimental data for the residual particle height $h$ after impact.

In Figure 5, the yield strength calculated from the experimental data is plotted depending on the ice fraction obtained from the supercooling of the drop with Eq. (1). As shown in the figure, for higher ice fractions, the experiments reveal a clear tendency towards higher values of $Y$. For purposes of implementing this relation in simulation codes, an empirical correlation between the ice fraction and the yield strength in the form of a power law has been computed and is 


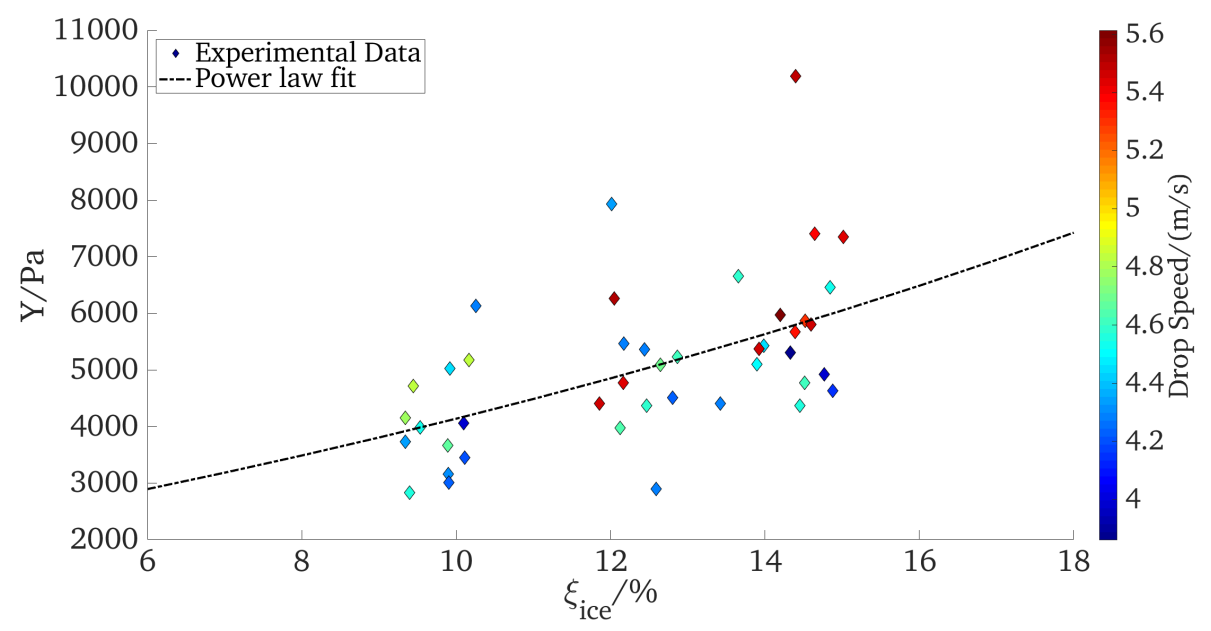

Figure 5. Yield strength determined from the experimental results for residual height plotted over the ice fraction of the corresponding mushy particle.

shown in Figure 5. This empirical function is given as

$$
Y\left(\xi_{\text {ice }}\right) \approx 4714 \mathrm{e}^{0.0046} \xi_{\text {ice }}-3309 \text {. }
$$

The general scatter of the data indicates significant uncertainty in the exact value of $Y$ and a larger amount of data would contribute to improvement of the predictive capability of this correlation. This uncertainty possibly originates from local imperfections in the crystalline ice structure or horizontal effects in the flow caused by stagnation of the ice/water mixture. Both effects are not accounted for in the model, although they might influence the development of the residual height of the ice cone, which is crucial for the determination of $Y$. Nevertheless, the present data presents a novel step in understanding the physics and dynamics related to the impact of supercooled drops following the dendritic freezing phase.

Next to the overall increase of yield strength for increasing ice fractions, the mushy particle impact speed, denoted by symbol colour in Figure 5, appears also to result in increasing yield strength. This trend is less distinctive from the figure, but a strain rate dependent yield strength as reported for pure ice in [19] could explain this velocity dependence in the data. Further systematic measurements are necessary to describe the rheology of the mushy particles more accurately and to determine the relative importance of other potential influences like viscous effects associated with the relative flow of the dispersed phase, etc.

\section{Conclusions}

The present study is devoted to the impact of dendritic frozen drops onto a cold surface. The performed experiments involved mushy particles originating from supercooled water drops in the temperature range from -11.5 to $-7^{\circ} \mathrm{C}$. After the dendritic solidification phase, the initial supercooling leads to ice fractions between $\xi_{\text {ice }}=9$ to $15 \%$. The observations obtained from high-speed recordings show a significant change of impact dynamics in comparison to a liquid drop. During its impact, the mushy particle exhibits characteristics of plastic flow. Hence, the ice/water mixture remaining on the surface forms a cone shape, whose apex height is characteristic for the yield strength $Y$ of its mushy phase. An existing model for the impact of a semi-brittle ice particle is adapted and used to calculate a corresponding yield strength from the residual cone height. The resulting yield strength obtained for the examined mushy particle exhibits an increase for increasing amount of ice in the mushy particle. On the basis of these outcomes, a semi-empirical model for the yield strength $Y=Y\left(\xi_{\text {ice }}\right)$ is determined. The proposed correlation and the findings of this study can aid in simulating the impact of mushy particle and advance the understanding of ice accretion due to impact of supercooled drops. 


\section{Acknowledgements}

The authors gratefully acknowledge the financial support of the Deutsche Forschungsgemeinschaft in the framework of the Collaborative Research Center SFB-TRR 75 "Droplet Dynamics Under Extreme Ambient Conditions", (TP C03, project number 84292822).

\section{Nomenclature}

$a(t) \quad$ Particle impression radius $/ \mathrm{m}$

$b(t) \quad$ Particle centre position $/ \mathrm{m}$

$c_{p} \quad$ Heat capacity $/\left(\mathrm{J} \mathrm{kg}^{-1} \mathrm{~K}^{-1}\right)$

$D_{0} \quad$ Initial mushy particle diameter $/ \mathrm{m}$

$h(t) \quad$ Particle height $/ \mathrm{m}$

$L \quad$ Latent heat of fusion (water) $/ \mathrm{J}$

$R_{0} \quad$ Initial particle radius $/ \mathrm{m}$

$t \quad$ Time $/ \mathrm{s}$

$U_{0} \quad$ Initial particle velocity $/\left(\mathrm{m} \mathrm{s}^{-1}\right)$

$\begin{array}{ll}u(t) & \text { Particle centre velocity } /\left(\mathrm{m} \mathrm{s}^{-1}\right) \\ V(t) & \text { Particle volume } / \mathrm{m}^{3} \\ Y & \text { Yield strength } / \mathrm{Pa} \\ \delta & \text { Dimensionless displacement } /- \\ \delta_{\max } & \text { Maximum displacement } /- \\ \Delta T_{0} & \text { Initial supercooling } / \mathrm{K} \\ \rho & \text { Density } /\left(\mathrm{kg} \mathrm{m}{ }^{-3}\right) \\ \xi_{\text {ice }} & \text { Mass fraction of ice } /-\end{array}$

\section{References}

[1] Cebeci, T., Kafyeke, F., 2003, Annual Review of Fluid Mechanics, 35 (1) pp. 11-21.

[2] Jasinski, W. J., Noe, S. C., Selig, M. S., Bragg, M. B., 1998, Journal of Solar Energy Engineering, (120) pp. 60-65.

[3] Szilder, K., Lozowski, E. P., Reuter, G., 2002, Journal of Offshore Mechanics and Arctic Engineering, (124) pp. 162-168.

[4] Makkonen, L., 1987, Cold Regions Science and Technology, (14) pp. 163-171.

[5] Yarin, A. L., 2006, Annual Review of Fluid Mechanics, 38 (1) pp. 159-192.

[6] Josserand, C., Thoroddsen, S. T., 2016, Annual Review of Fluid Mechanics, 48 (1) pp. 365-391.

[7] Yarin, A. L., Roisman, I. V., Tropea, C., 2017, Collision Phenomena in Liquids and Solids, Cambridge University Press.

[8] Jung, S., Tiwari, M. K., Doan, N. V., Poulikakos, D., 2012, Nature communications, 3 (1) pp. 1-8.

[9] Schremb, M., Tropea, C., 2016, Physical Review. E, 94 (5-1) p. 052804.

[10] Roisman, I. V., Tropea, C., 2020, Current Opinion in Colloid \& Interface Science p. 101400.

[11] Schremb, M., Roisman, I. V., Tropea, C., 2017, Physical Review. E, 95 (2-1) p. 022805.

[12] Blanken, N., Saleem, M. S., Thoraval, M.-J., Antonini, C., 2021, Current Opinion in Colloid \& Interface Science, 51 p. 101389.

[13] Marston, J. O., Mansoor, M. M., Thoroddsen, S. T., 2013, Physical Review. E, Statistical, Nonlinear, and Soft Matter Physics, 88 (1) p. 010201.

[14] Kant, P., Müller-Groeling, H., Lohse, D., 2020, Physical Review Letters, 125 (18) p. 184501.

[15] Roisman, I. V., Tropea, C., 2015, Proceedings of the Royal Society A: Mathematical, Physical and Engineering Sciences, 471 (2183) p. 20150525.

[16] Huppert, H. E., 1990, Journal of Fluid Mechanics, 212 pp. 209-240.

[17] Worster, M. G., 1992, The Dynamics of Mushy Layers, Springer Netherlands, Dordrecht, pp. 113-138.

[18] Schremb, M., 2018, Hydrodynamics and Thermodynamics of Ice Accretion through Impact of Supercooled Large Droplets: Experiments, Simulations and Theory, Ph.D. thesis, Technische Universität Darmstadt, Darmstadt.

[19] Tippmann, J. D., Kim, H., Rhymer, J. D., 2013, International Journal of Impact Engineering, 57 (7) pp. 43-54. 